rarely encountered despite platelet counts of $10 \times 10^{9} / \mathrm{L}$ or less in 11 cases, $20 \mathrm{x}$ $10^{9} / \mathrm{L}$ or less in 67 , and $50 \times 10^{9} / \mathrm{L}$ or less in 306 . Among a total of 5223 LPs performed either at diagnosis or during a median of 4 L.Ps for intrathecal chemotherapy (methotrexate, hydrocortisone, cytarabine), the estimated probabilities of serious complications (95\% confidence intervals) in relation to platelet count (pc x $10^{9} / \mathrm{L}$ ) were $0-40.19 \%$ with $1-5 \mathrm{pc}, 0-13.21 \%$ with $6-10 \mathrm{pc}, 0$ $2.05 \%$ with $11-20 \mathrm{pc}$, and $0-0.10 \%$ with $>100 \mathrm{pc}$. Traumatic LP recorded in 548 procedures $(10.5 \%)$ was not associated with adverse sequelae. LPs preceded by prophylactic platelet transfusion $(n=167)$ and without post-transfusion platelet counts were excluded. LPs were performed by pediatric oncologists, pediatric oncology fellows, pediatric residents, and nurse practitioners. Only 29 LPs were

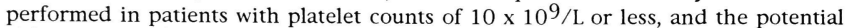
risks associated with LP without platelet transfusion in this group of patients is not determined. (Howard SC, Gajjar A, Ribeiro RC et al. Safety of lumbar puncture for children with acute lymphoblastic leukemia and thrombocytopenia. IAMA November 1, 2000;284:2222-2224). (Reprints: Scott C Howard MD, Department of Hematology-Oncology, ALSAC Bldg, Room C6005, St Jude Children's Research Hospital, $332 \mathrm{~N}$ Lauderdale St, Memphis, TN 38105).

COMMENT. In the management of children with acute lymphoblastic leukemia, diagnostic and therapeutic lumbar puncture (LP) procedures may be performed without risk of serious hemorrhagic complication despite thrombocytopenia. Prophylactic platelet transfusion is not advised prior to LP when platelet counts are higher than $10 \times 10^{9} / \mathrm{L}$. Below this number of platelets, the safety of LP is unproven. An article entitled "the perils of platelet transfusions" (Kruskall MS. N Engl I Med 1997;337:1914-1915) is cited as a contraindication to platelet transfusion as a routine practice.

Consultation with my colleagues in the Division of Hematology/Oncology at Children's Memorial Hospital, Chicago, corroborates these findings and practice. Platelet transfusion is not routinely administered prior to LP. Furthermore, no serious hemorrhagic complication has been encountered even with platelet counts of $3-4 \times 10^{9} / \mathrm{L}$. The majority of patients undergoing LP on this service receive a short-acting sedative and a topical application of lidocaine cream (Emla $\left.{ }^{\circledR}\right)$, since the risks of potential hemorrhage may be increased in patients requiring excessive restraint. (personal communication)

\title{
SUBDURAL HEMATOMA AND GLUTARIC ACIDURIA TYPE 1
}

An 8-week-oid male infant diagnosed with bilateral subdural hematoma following a reported fall and head injury was found to have glutaric aciduria type 1 and was subsequently treated by dietary modification at Addenbrooke's Hospital, Cambridge, UK. Initially suspected to have a nonaccidental injury, the infant had been placed in foster care and the mother prosecuted. The metabolic diagnosis was suspected at 6 months of age when the infant presented with macrocephaly and developmental delay. Urine organic analysis showed elevated excretion of glutaric acid and 3-hydroxyglutaric acid. Glutaryl-CoA dehydrogenase activity was absent in cultured fibroblasts. Despite reduced lysine/tryptophan diet with carnitine supplement, the infant remains globally retarded. (Hartley LM, Khwaja OS, Verity CM. Glutaric aciduria type 1 and nonaccidental head injury. Pediatrics Jan 2000;107:174-176). (Reprints: Dr CM Verity, Box 181, Addenbrooke's Hospital, Cambridge CB1 2QQ UK.

COMMENT. Infants with subdural hematoma and suspected NAI should receive metabolic screening before parental charges are pursued. 